\title{
The Field Modulation Effect of a Fluoride Plasma Treatment on the Blocking Characteristics of AIGaN/ GaN High Electron Mobility Transistors
}

\author{
Young-Shil Kim, O-Gyun Seok, and Min-Koo Han ${ }^{\dagger}$ \\ School of Electrical Engineering, Seoul National University, Seoul 151-744, Korea \\ Min-Woo Ha \\ Korea Electronics Technology Institute, Seongnam 463-81, Korea
}

Received May 9, 2011; Revised May 20, 2011; Accepted June 2, 2011

\begin{abstract}
We designed and fabricated aluminium gallium nitride (AlGaN)/GaN high electron mobility transistors (HEMTs) with stable reverse blocking characteristics established by employing a selective fluoride plasma treatment on the drainside gate edge region where the electric field is concentrated. Implanted fluoride ions caused a depolarization in the AlGaN layer and introduced an extra depletion region. The overall contour of the depletion region was expanded along the drift region. The expanded depletion region distributed the field more uniformly and reduced the field intensity peak. Through this field modulation, the leakage current was reduced to $9.3 \mathrm{nA}$ and the breakdown voltage $\left(\mathrm{V}_{\mathrm{BR}}\right)$ improved from $900 \mathrm{~V}$ to $1,400 \mathrm{~V}$.
\end{abstract}

Keywords: Gallium nitride, Aluminium gallium nitride, High electron mobility transistor, Breakdown, Fluoride

\section{INTRODUCTION}

Aluminium gallium nitride (AlGaN)/GaN high electron mobility transistors (HEMTs) are a promising candidate for high power and high frequency applications due to their superior material properties [1,2]. Leakage current and premature breakdown not only degrade the blocking capabilities of power devices but also cause power losses in the system [3]. The active region leakage current is attributed to the electrons injected through the rectifying contact. In a lateral power device, the electron injection is mainly due to the strong electric field concentrated in the depletion region under the gate electrode compared to other factors [4]. In order to achieve stable blocking characteristics,

${ }^{\dagger}$ Author to whom all correspondence should be addressed:

E-mail:mkh@snu.ac.kr

Copyright $\odot 2011$ KIEEME. All rights reserved.

This is an open-access article distributed under the terms of the Creative Commons Attribution Non-Commercia
License (http://creativecommons.org/licenses/by-nc/3.0) which permits unrestricted noncommercial use,

Listribution, and reproduction in any medium, provided the original work is properly cited.
ditiction such as low leakage current and high breakdown voltage, the peak intensity of the electric field at the gate depletion needs to be reduced. Our work concentrated on an investigation into the field modulation effect caused by implanted fluoride ions as well as the influence of the modified internal field on the blocking characteristics of an AlGaN/GaN HEMT. An improved blocking capability was confirmed by the measured leakage current and the breakdown voltage of the fabricated device.

\section{DEVICE STRUCUTURE AND FABRICATION}

AlGaN/GaN heterostructure was grown on SiC substrates using metal organic chemical vapor deposition. A $30 \mathrm{~nm}$-thick unintentionally doped $\mathrm{Al}_{0.3} \mathrm{Ga}_{0.7} \mathrm{~N}$ and $\mathrm{n} 3 \mu \mathrm{m}$-thick GaN buffer formed the two dimensional electron gas (2DEG) channel of the AlGaN/GaN HEMT. An undoped GaN capping layer was then grown. A mesa structure with the thickness of $140 \mathrm{~nm}$ was formed for device isolation using the inductively coupled plasma 


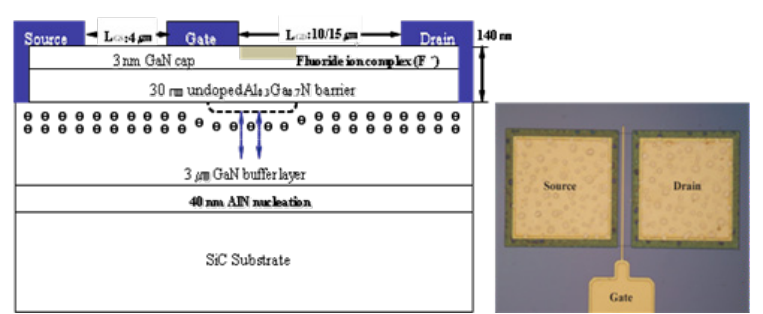

Fig. 1. The cross-sectional view and microscopic image of the fabricated AlGaN/GaN high electron mobility transistor.

reactive ion etching (RIE) apparatus. In order to create the source and drain electrodes, a Ti/Al/Ni/Au (20/80/20/100 nm) based ohmic contact was e-gun evaporated then annealed at $870^{\circ} \mathrm{C}$ for 30 seconds under $\mathrm{N}_{2}$ ambient. A Ni/Au $(30 / 150 \mathrm{~nm})$ based Schottky contact was also e-gun evaporated. The standard lift off method was used to define these metallization patterns. Crosssectional view of the fabricated device was shown in Fig. 1.The device was then annealed at $500^{\circ} \mathrm{C}$ for 5 minutes under $\mathrm{O}_{2}$ and $\mathrm{N}_{2}$ ambient. A selective fluoride plasma treatment using a capacitive coupled plasma RIE apparatus was performed on the drainside gate edge region with selected radio frequency (RF) power conditions (from 15 to $60 \mathrm{~W}$ ) for 120 seconds. The flow rate of the $\mathrm{CF}_{4}$ gas was set to $20 \mathrm{sccm}$ at a pressure of $50 \mathrm{mT}$. The plasma treatment condition used for the device fabrication was greatly attenuated in order to minimize the surface damage. Through auger electron spectroscopy (AES) measurement, it was confirmed that fluoride atoms were implanted into the AlGaN layer.

\section{RESULTS AND DISCUSSION}

Fluoride atoms were implanted into AlGaN barrier layer through soft etching. The depth of the fluoride atoms was controlled by the plasma power to not invade the channel. As shown in Fig. 2, the fluoride atoms were distributed to within $5 \mathrm{~nm}$ from the AlGaN surface, considering the profiling time of the AES measurement. Most of these atoms were detected at the surface of the AlGaN layer. The concentration of fluoride ions decreased rapidly along the depth of the device.

The fluoride atoms implanted into barrier layer performed as negatively charged ions due to their strong electron affinity [57]. The implanted fluoride ions inhibited polarization charges from being induced at the AlGaN surface (negative polarization charge, $-\rho_{\mathrm{PZ}}$ ) and at the $\mathrm{AlGaN} / \mathrm{GaN}$ interface (positive polarization charge, $+\rho_{\mathrm{PZ}}$ ) by an electrostatic interaction with the identical dipole arrays in the $\mathrm{AlGaN}$ layer.

Generally in the AlGaN layer, the polarization field is induced by the negative and positive polarization charges localized at both sides of the AlGaN layer. The polarization charges are introduced by the heads and the tails of the dipole array. The polarization is reinforced by a strain (tensile stress) in the AlGaN layer, whereas the polarization in the GaN layer is not. The discrepancy in the polarization is reflected in the difference of the internal field between the $\mathrm{AlGaN}$ and GaN layer. Due to this difference in the internal field, an electron channel (or 2DEG) is formed at the interface to offset the discrepancy [8,9]. In the plasma treated AlGaN layer, however, the negatively charged fluoride ions prevented the anions and cations from forming an electric dipole system through an electric repulsion force. A stack of disrupted dipole arrays has great difficulty in inducing the polarization charges at both ends of the stack. The polarization field in the plasma treated $\mathrm{AlGaN}$ layer was smaller than that found in a conventional HEMT. The difference in the field between the barrier

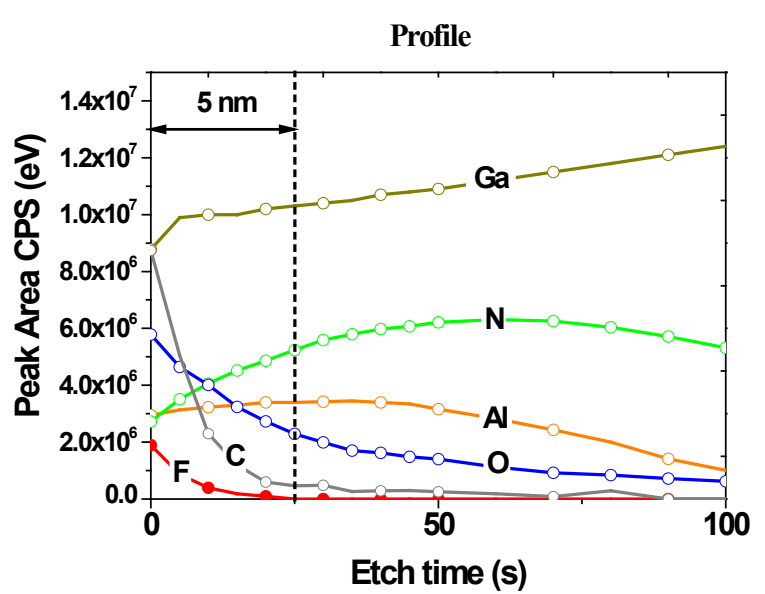

Fig. 2. The auger electron spectroscopy measurement results of the fluoride plasma treated $\mathrm{AlGaN} / \mathrm{GaN}$ high electron mobility transistor at the radio frequency power of $30 \mathrm{~W}$.

and buffer layer was also reduced. The electrons induced to form an equilibrium state (charge neutrality condition) at the AlGaN/ GaN interface were decreased. The discrepancy of the field between the two layers was inversely proportional to the RF power. In the case of hard plasma treatment, where high RF power and long process times are used, there is no need for the electrons to be induced at the interface to compensate for the discrepancy. As a result, the channel of the AlGaN/GaN HEMT fabricated with a hard plasma treatment is fully depleted. The device operates in the enhancement-mode.

In this experiment, however, a soft plasma treatment was used in order to minimize the loss in the forward current and change the depletion region to be suitable for a stable blocking capability. The degree of the field modulation in the barrier layer was elaborately controlled by minute RF variations (15/30/45/60 W). In the soft plasma treated device, the polarization field was moderately modulated. This not only partially depletes the carriers in the channel but makes the depletion region more extended than before. A series of vertically expanded depletion regions was formed along the drift region by the fluoride plasma treatment. The expanded depletion region induced by the plasma treatment made the whole contour of the depletion region near the gate electrode sufficiently enlarged to sustain a high reverse bias.

The modification of the internal field and depletion contour was confirmed by an upward band bending in the simulated energy band structure (Fig. 3) [10].

As shown in Fig. 3(a), the gradient of the potential (energy band) of the plasma treated device is relatively shallow at the $\mathrm{AlGaN}$ and GaN region, whereas that of the conventional one is steep at both regions. According to the Poisson equation, the gentle gradient of the potential indicates that a low electric field is induced at both layers. As the slope of the potential at both regions become more gradual, the depth of the quantum well formed at interface becomes shallow. It is directly related to the decrease of the carrier density, as shown in Fig. 3(b). In addition, the expansion of the depletion region in the $\mathrm{GaN}$ is confirmed in the simulated band structure. The depletion which plays a significant role in the reverse operation mode is the one formed in the GaN buffer layer rather than that formed in the AlGaN layer. In $\mathrm{AlGaN} / \mathrm{GaN}$ HEMTs, it is defined as the length from the interface and the plateau of the band. As shown in Fig. 3(a), the depletion depth of the proposed device is much deeper than that of a conventional HEMT without any reverse bias.

The change of the depletion was verified from the measured 
(a)

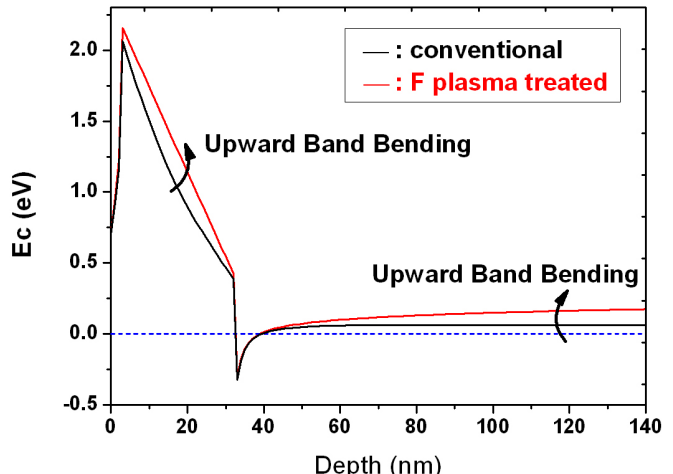

(b)

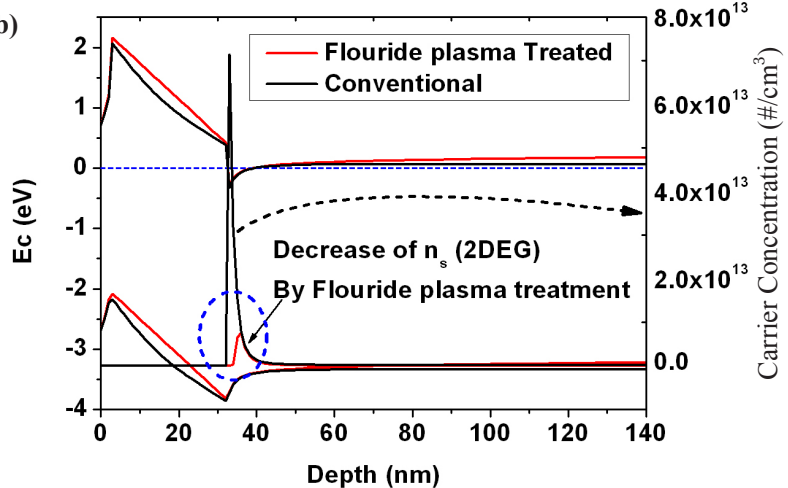

Fig. 3. The simulated energy bands of the fluoride plasma treated AlGaN/GaN high electron mobility transistor: (a) conduction band, (b) the entire band structure and channel carrier density.

C-V characteristic (Fig. 4), particularly from the measured offstate gate drain capacitance. For ease of understanding, a simplified equivalent capacitance model was used. The equivalent capacitance between the gate and drain can be simplified as a parallel connection between the capacitance of the capping layer and the series of the two AlGaN capacitances and the channel capacitance. The approximate equation for the gate-drain capacitance is:

$$
C_{G D}=C_{(c a p)}+C_{c h} C_{A l G a N} /\left(2 C_{c h}+C_{A l G a N}\right)
$$

Under a reverse bias condition, the gate bias is lowered below the pinch-off voltage. The channel capacitance $\left(\mathrm{C}_{\mathrm{ch}}\right)$ is equivalent to the depletion capacitance $\left(\mathrm{C}_{\mathrm{dep}}\right)$. Eq. (1) can therefore be simplified. The resulting equation for the off-state gate drain capacitance under blocking mode is:

$$
C_{\text {OFF }}=C_{(c a p)}+C_{\text {dep }} \text {, where } C_{\text {dep }}=\varepsilon_{\text {GaN }} / t_{\text {dep }} \cdot W \cdot L_{\text {exp }}
$$

In Eq. (2), the capacitance of the GaN capping layer $\left(\mathrm{C}_{\text {cap }}\right)$ is fixed. The gate drain capacitance entirely depends on the depletion capacitance.

The area exposed to the plasma treatment was increased from 100 to $200 \mathrm{\mu m}^{2}$; the off-state capacitance was increased from 0.119 to $0.160 \mathrm{pF}$. The increase in the off-state capacitance indicates that the area of the depletion capacitor was increased. In terms of the device, it means that a lateral expansion of the depletion occurred. The dilated depletion alleviated the field concentration at the gate depletion by distributing the electric field flux into an extra depletion region induced by the plasma treatment. Consequently, the electric field caused by a reverse bias was spread more uniformly in the drift region than before. The field intensity at the unit area of the gate depletion was thereby decreased.

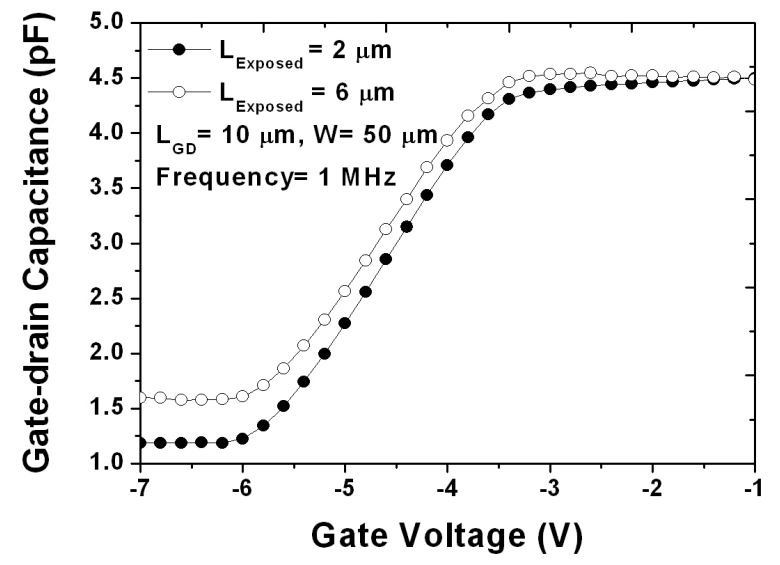

Fig. 4. The measured off-state gate-drain capacitance of the fluoride plasma treated AlGaN/GaN high electron mobility transistor.

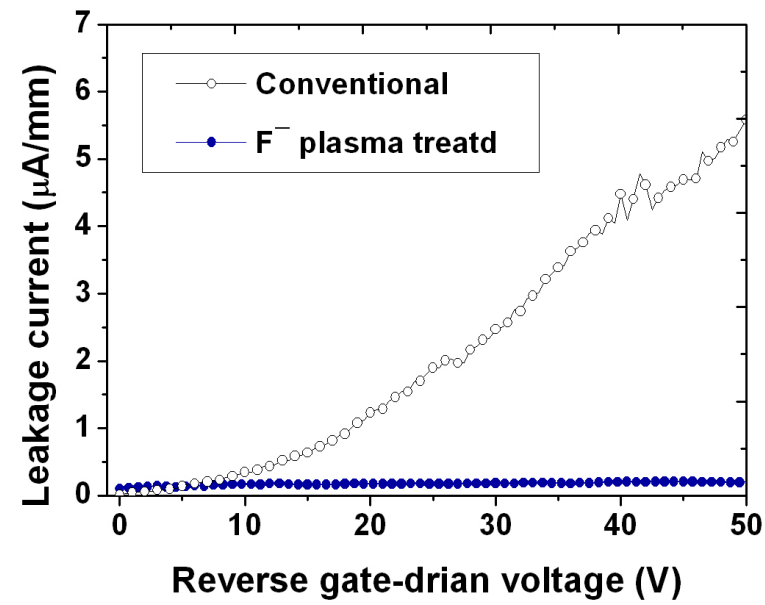

Fig. 5. The measured leakage current of the plasma treated AlGaN/ GaN high electron mobility transistor.

The leakage current was measured under the reverse bias condition where the gate and drain voltage was $-7 \mathrm{~V}$ and $50 \mathrm{~V}$, respectively. As field intensity at the gate depletion reduced, the probability that electrons would be injected into the barrier layer was decreased. The suppression of the electron injection led to a decrease in the leakage current. As shown in Fig. 5, the leakage current of the untreated device was $0.28 \mu \mathrm{A}$ (current density: 5.6 $\mu \mathrm{A} / \mathrm{mm}$ ), whereas that of the plasma treated HEMT was $9.5 \mathrm{nA}$ (current density: $190 \mathrm{nA} / \mathrm{mm}$ ).

The breakdown characteristics of the fluoride plasma treated AlGaN/GaN HEMT was also investigated. As shown in Fig. 6, the breakdown of the conventional device occurred at $900 \mathrm{~V}$; it showed soft breakdown characteristics. The breakdown of plasma treated device (at an RF power of $30 \mathrm{~W}$ ) occurred at 1,400 V; it showed hard breakdown characteristics where leakage current was suppressed effectively right up to the breakdown regardless of reverse bias. It was reflected in both the suppression of leakage current and the improvement of breakdown voltage that the field modulation caused by the fluoride plasma treatment dropped the peak intensity of the electric field by introducing an additional depletion region near the gate electrode. The reliable blocking characteristics obtained by the fluoride plasma treatment guarantees a high power-handling capability for the device and a small power loss in the system.

The blocking capability of the plasma treated device was im- 


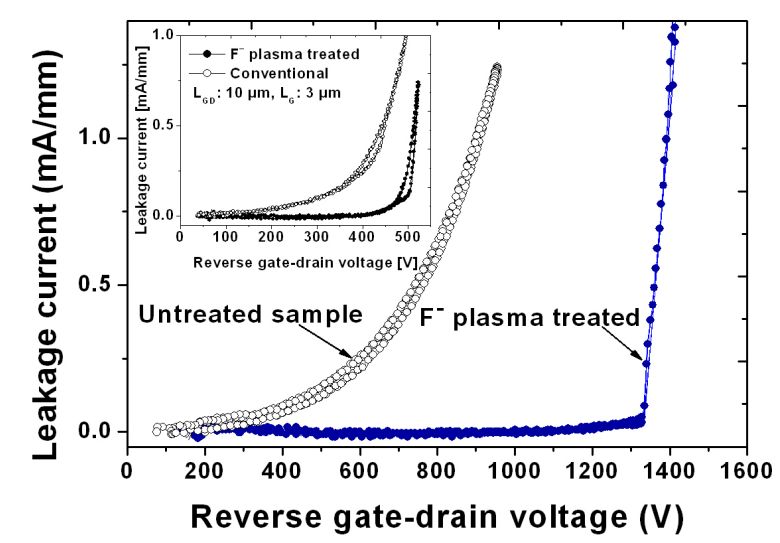

Fig. 6. The measured breakdown characteristics of the fluoride plasma treated $\mathrm{AlGaN} / \mathrm{GaN}$ high electron mobility transistor (at $30 \mathrm{~W}$ ).

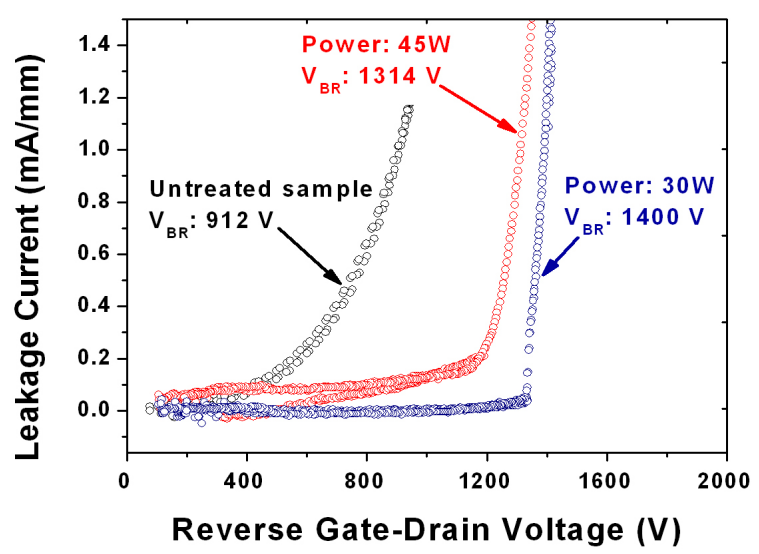

Fig. 7. The dependency of the breakdown characteristics on the plasma radio frequency power.

proved in proportion to the RF power until $30 \mathrm{~W}$ (Fig. 7). At RF powers higher than $30 \mathrm{~W}$, however, the blocking characteristic was degraded in proportion to the RF power increase. Although the breakdown voltage of the device plasma treatment at the power of $45 \mathrm{~W}$ was over $1300 \mathrm{~V}$, its reverse operation was more unstable than that found in the device plasma treated at $30 \mathrm{~W}$. The leakage current of the devices plasma treated at RF powers over $30 \mathrm{~W}$ was influenced by the reverse bias and relatively high. The leakage current dependency on the reverse bias increased as the RF power increased. The breakdown voltage of the device treated at $60 \mathrm{~W}$ was similar to that of a conventional HEMT (not shown in Fig. 7). The leakage current was slightly higher than that of a conventional HEMT. At the plasma power over $30 \mathrm{~W}$, the side effects caused by the plasma treatment outweighed the field modulation effects. To be more specific, at a high plasma power, the surface leakage current due to plasma damage overwhelmed the stable blocking operation established by the field modulation effect. Therefore, the device failed to operate stably in the reverse blocking mode irrespective of the field modulation effect.

\section{CONCLUSIONS}

We investigated the effect of the field modulation effect caused by implanted fluoride ions as well as the influence of the modified internal field on the blocking characteristics of $\mathrm{AlGaN} / \mathrm{GaN}$ HEMTs. The fluoride plasma treatment expanded the gate depletion region laterally. This reduced the peak of the electric field concentrated at the gate edge by distributing the electric field more uniformly. Through this mechanism, the fluoride plasma treated device achieved a high breakdown voltage. The measured breakdown voltage of the plasma treated AlGaN/GaN HEMT was $1.4 \mathrm{kV}$. It was higher than that found for an untreated device by $55 \%$.

\section{ACKNOWLEDGMENTS}

This work was supported by a 'Power Generation \& Electricity Delivery' department of the Korea Institute of Energy Technology Evaluation and Planning (KETEP) grant funded by the Korea Government Ministry of Knowledge Economy.

\section{REFERENCES}

[1] A. Chini, D. Buttari, R. Coffie, S. Heikman, S. Keller, and U. K. Mishra, Electron. Lett 40, 73 (2004) [DOI: 10.1049/el:20040017].

[2] Y. F. Wu, A. Saxler, M. Moore, R. P. Smith, S. Sheppard, P. M. Chavarkar, T. Wisleder, U. K. Mishra, and P. Parikh, IEEE Electron Device Lett. 25, 117 (2004) [DOI: 10.1109/LED.2003.822667].

[3] A. Khaligh and A. Emadi, J. Electr. Eng. Technol. 1, 63 (2006).

[4] X. Huili, Y. Dora, A. Chini, S. Heikman, S. Keller, and U. K. Mishra, IEEE Electron Device Lett. 25, 161 (2004) [DOI: 10.1109/ LED.2004.824845].

[5] C. Yong, Z. Yugang, K. J. Chen, and K. M. Lau, IEEE Electron Device Lett. 26, 435 (2005) [DOI: 10.1109/LED.2005.851122].

[6] L. Yuan, M. Wang, and K. J. Chen, Physica Status Solidi (c) 6, S944 (2009) [DOI: 10.1002/pssc.200880776]

[7] F. Medjdoub, M. Alomari, J. F. Carlin, M. Gonschorek, E. Feltin, M. A. Py, C. Gaquiere, N. Grandjean, and E. Kohn, Electron. Lett 44, 696 (2008) [DOI: 10.1049/el:20080864].

[8] O. Ambacher, J. Smart, J. R. Shealy, N. G. Weimann, K. Chu, M. Murphy, W. J. Schaff, L. F. Eastman, R. Dimitrov, L. Wittmer, M. Stutzmann, W. Rieger, and J. Hilsenbeck, J. Appl. Phys. 85, 3222 (1999) [DOI: 10.1063/1.369664].

[9] F. Sacconi, A. Di Carlo, P. Lugli, and H. Morkoc, IEEE Trans. Electron Dev. 48, 450 (2001) [DOI: 10.1109/16.906435].

[10] Yong Cai, Yugang Zhou, Lau, K.M. and Chen, K.J, IEEE Trans. Electron Dev. 53, 2207 (2006) [DOI: 10.1109/TED.2006.881054]. 